\title{
DEVELOPMENT OF RITONAVIR LOADED NANOPARTICLES: IN VITRO AND IN VIVO CHARACTERIZATION PRAVIN S PATIL ${ }^{1,2 *}$, SHASHIKANT C DHAWALE ${ }^{3}$
}

\begin{abstract}
${ }^{1}$ Department of Pharmaceutics, Ashokrao Mane College of Pharmacy, Peth Vadgaon, Kolhapur, Maharashtra, India. ${ }^{2}$ Department of Biopharmaceutics, Government College of Pharmacy, Karad, Maharashtra, India. ${ }^{3}$ Department of Pharmacology, School of Pharmacy, Swami Ramanand Teerth Marathwada University, Nanded, Maharashtra, India. Email: pspatil1812@gmail.com
\end{abstract}

Received: 16 October 2017, Revised and Accepted: 09 December 2017

\section{ABSTRACT}

Objective: The purpose of the present investigation was to develop a nanosuspension to improve dissolution rate and oral bioavailability of ritonavir.

Methods: Extended-release ritonavir loaded nanoparticles were prepared using the polymeric system by nanoprecipitation technique. Further, the effect of Eudragit RL100 (polymeric matrix) and polyvinyl alcohol (surfactant) was investigated on particle size and distribution, drug content, entrapment efficiency, and in vitro drug release from nanosuspension where a strong influence of polymeric contents was observed. Drug-excipient compatibility and amorphous nature of drug in prepared nanoparticles were confirmed by Fourier transform infrared spectroscopy, differential scanning calorimetry, and powder X-ray diffraction studies, respectively.

Results: Hydrophobic portions of Eudragit RL100 could result in enhanced encapsulation efficiency. However, increase in polymer and surfactant contents lead to enlarged particle size proportionately as confirmed by transmission electron microscopy. Nanosuspension showed a significant rise in dissolution rate with complete in vitro drug release as well as higher bioavailability in rats compared to the pure drug.

Conclusion: The nanoprecipitation technique used in present research could be further explored for the development of different antiretroviral drug carrier therapeutics.

Keywords: Ritonavir, Nanoparticle, Nanoprecipitation, Lyophilization, In vitro dissolution study, In vivo bioavailability study.

(c) 2018 The Authors. Published by Innovare Academic Sciences Pvt Ltd. This is an open access article under the CC BY license (http://creativecommons. org/licenses/by/4. 0/) DOI: http://dx.doi.org/10.22159/ajpcr.2018.v11i3.23145

\section{INTRODUCTION}

The fundamental events are controlling drug absorption after oral administration is its solubility and dissolution in the gastrointestinal milieu as well as permeability through the membrane [1-3]. Solubility and dissolution rate of drug could be enhanced with use of cosolvents or salt/prodrug formation and different approaches such as solid dispersion, cyclodextrin, melt extrusion, emulsion, microemulsion, liposome and micellar system, addition of surfactant, microenvironmental $\mathrm{pH}$ change, hydrotropic solubilization of drug, and crystal engineering (solvates, polymorphs, and cocrystals) [411]. Moreover, use of particle size reduction approaches to form stable nanometer-sized drug nanosuspension or nanoparticles is a relatively new formulation strategy for solubility and dissolution enhancement $[12,13]$.

Nanosuspension is one of the preferred physical systems as the components therein readily form a solution when getting in contact with a liquid medium such as gastric juice. The ease of dissolution by an amorphous substance from nanosuspension was attributed to its high energy content compared to pure components in the crystalline form. This system could provide a strategy to achieve therapeutic efficacy by improving drug dissolution at the site of administration. The nanoprecipitation or solvent displacement technique is easy, rapid one-step process with low complexity which gives nanoparticles instantly $[14,15]$. Briefly, it requires two miscible solvents, out of which in any one solvent (good solvent) both the drug and polymer should get solubilized easily while the second solvent act as a bad or antisolvent. Addition of the polymer solution to the antisolvent causes diffusion and dispersion of the polymer into the dispersing medium with rapid desolvation of the polymer that leads to nanoprecipitation followed with immediate drug entrapment.

Protease inhibitors used in the treatment of AIDS found to influence the glycoprotein synthesis independently which in turn inhibits the growth of HIV. Ritonavir, one of the potential protease inhibitor could also act as a substrate for efflux pump that is ultimately preventing the elimination of other coadministered antiretroviral drugs. The solubility limited bioavailability of ritonavir (biopharmaceutical classification system Class II) could be enhanced through improving its solubility in the gastric fluid by preparation of solid dispersion [14-16].

The purpose of the present study is to develop a nanosuspension of ritonavir by the nanoprecipitation technique with improved dissolution rate and hence oral bioavailability. The effect of Eudragit RL100 (ERL100, a polymeric matrix) and polyvinyl alcohol ([PVA], surfactant) contents was also studied on the quality attributes of the prepared nanosuspension such as particle size, entrapment efficiency, in vitro dissolution, and in vivo bioavailability.

\section{METHODS}

\section{Materials}

Ritonavir was supplied by Macleod Pharmaceutical Pvt., Ltd., Mumbai (Maharashtra, India) as a gift sample. ERL100 and PVA were kindly gifted by Degussa Pharma. Pvt. Ltd., Mumbai (Maharashtra, India). All other chemicals used were of analytical grade (Loba Chemie, Mumbai, Maharashtra, India). Triple distilled water was used throughout the experimentation.

\section{Methods}

Preparation of nanosuspension and nanoparticles

Nanosuspension of ritonavir was prepared by nanoprecipitation technique [16]. Initially, ERL100 and ritonavir (100 mg) were dissolved in methanol $(25 \mathrm{ml})$ with the aid of sonication. The resulting solution was slowly injected in aqueous solution (30 ml) of PVA kept under continuous stirring at $2000 \mathrm{rpm}$. The stirring was further continued for $2 \mathrm{~h}$ at $40^{\circ} \mathrm{C}$ to allow complete evaporation of methanol that resulted in the formation of nanosuspension. The contents of ERL100 and PVA were identified as the independent variables affecting the drug content 
and particle size (dependent variables, Table 1). Further, ritonavir nanoparticles were obtained by lyophilization of the optimized batch of nanosuspension at $-42^{\circ} \mathrm{C}$ for $72 \mathrm{~h}$ using laboratory freeze dryer (Khera Instruments, Mumbai, India).

\section{Characterization of nanoparticles}

Particle size analysis

The ritonavir and nanoparticles were dispersed in distilled water and the average particle size was determined by Malvern Master Sizer 2000 (Malvern Instruments, UK).

\section{Drug content}

A specified quantity of nanoparticles was dissolved in methanol and the resulting solution was analyzed using ultraviolet (UV)-visible spectrophotometer (Shimadzu 1800, Japan) for estimation of drug content $[4,11]$.

\section{Entrapment efficiency}

Unentrapped ritonavir was separated from the nanosuspension by centrifugation (Eppendorf 5810R, Germany) at $14000 \mathrm{rpm}$ for $2 \mathrm{~h}[4,11]$ and used for the estimation of encapsulation efficiency of ritonavir in prepared nanosuspension.

\section{Fourier transform infrared spectroscopy (FTIR) analysis}

FTIR spectra of ritonavir and nanoparticles (lyophilized nanosuspension) were recorded using FTIR spectrometer (Shimadzu Japan) to ascertain any structural or chemical changes in drug during and after processing. Samples were prepared using $\mathrm{KBr}$ pellet method (1:100) and spectra were recorded within range of $4000-400 \mathrm{~cm}^{-1}$ at the resolution of $2 \mathrm{~cm}^{-1}$.

\section{Differential scanning calorimetry (DSC) study}

DSC thermograms of ritonavir and nanoparticles were recorded using DSC (Perkin Elmer Pyris Diamond DSC, Waltham, Massachusetts, US) calibrated with indium and zinc over a temperature range of $100^{\circ} \mathrm{C}-300^{\circ} \mathrm{C}$ at a heating rate of $10^{\circ} \mathrm{C} / \mathrm{min}$.

Powder X-ray diffraction (PXRD) study

PXRD patterns of the ritonavir and nanoparticles were recorded using X-ray diffractometer (PW 1729, Phillips, Netherland) within scanning range of $5^{\circ}-70^{\circ}(2 \theta)$. The sample was initially irradiated with monochromatized $\mathrm{Cu}-\mathrm{Ka}$ radiation $\left(1.542 \mathrm{~A}^{\circ}\right)$ and analyzed at a voltage of $30 \mathrm{kV}$ and current of $30 \mathrm{~mA}$.

\section{Transmission electron microscopy (TEM) study}

Morphological characteristics of ritonavir loaded nanoparticles were studied by TEM (JEM-1230, JOEL, Tokyo, Japan). It gives an idea about the integral matrix and shape of the individual particle. A few drops of suitably diluted ritonavir nanosuspension were placed on a carboncoated grid and kept aside for $5 \mathrm{~min}$ to allow better adsorption on the carbon film. Further, the excess solution was drained out with aid of a filter paper and a drop of phosphotungstic acid (1\%) was placed and images were captured.

Table 1: Polymer contents used in the preparation of ritonavir nanosuspension

\begin{tabular}{lll}
\hline Batch code & ERL100 $(\% \mathbf{w} / \mathbf{v})$ & PVA $(\% \mathbf{w} / \mathbf{v})$ \\
\hline RNP1 & 0.30 & -- \\
RNP2 & 0.45 & -- \\
RNP3 & 0.60 & -- \\
RNP4 & -- & 0.5 \\
RNP5 & -- & 1.0 \\
RNP6 & -- & 1.5 \\
\hline
\end{tabular}

PVA: Polyvinyl alcohol, RNP: Required navigation performance

\section{In vitro dissolution study}

An accurately weighed quantity of powder equivalent to $20 \mathrm{mg}$ of the drug from pure ritonavir, physical mixture, nanosuspension, and nanoparticles was tested separately for estimation of release kinetics using USP Type II dissolution test apparatus (Labindia 2000, Mumbai, Maharashtra, India). The dissolution medium used was $900 \mathrm{ml}$ of $0.1 \mathrm{~N}$ $\mathrm{HCl}$ maintained at $37 \pm 0.5^{\circ} \mathrm{C}$ under continuous stirring at $50 \mathrm{rpm}$. Aliquots $(5 \mathrm{ml})$ were withdrawn at predetermined time intervals and replaced with fresh dissolution medium to maintain a sink condition. The aliquots withdrawn were filtered through Whatman filter $(0.2 \mu \mathrm{m})$ and analyzed using UV-visible spectrophotometer (Shimadzu UV 1700, Japan) at $246 \mathrm{~nm}$ for estimation of the rate of ritonavir release.

\section{In vivo bioavailability study}

The in vivo study was performed for estimation and comparison of bioavailability of the drug in its pure form as well as nanoparticles. The protocol of the in vivo study in animals was approved by Institutional Animal Ethics Committee, and the experiments were performed as per committee for prevention, control, and supervision of experimental animals guidelines. The in vivo bioavailability of ritonavir in pure form (reference) and nanoparticle form (test) was assessed in Male Wistar rats $(200-250 \mathrm{~g})$ by selecting a parallel design comprising two groups with six rats in each group. Before $12 \mathrm{~h}$ of experimentation, the rats were fasted and further fed after $4 \mathrm{~h}$ of the dosing. The animals were having free access to the tap water throughout the experimentation. A suspension of pure ritonavir (reference) and ritonavir nanoparticles (test) prepared separately in distilled water was administered orally to the rats from the first and second group, respectively, at a dose of $10 \mathrm{mg} / \mathrm{kg}$. An aliquot $(0.5 \mathrm{ml})$ of blood sample was withdrawn from the retro-orbital vein at predosing $(0 \mathrm{~h})$ and post-dosing intervals at different time points $(0.25,0.5,1,2,3,4,8,12,24$, and $48 \mathrm{~h})$, collected into Eppendorf tubes and subsequently centrifuged at $8000 \mathrm{rpm}$ for $5 \mathrm{~min}$. The plasma was separated and transferred to respective Eppendorf tubes and stored at $-20^{\circ} \mathrm{C}$ for further estimation of plasma profile of the drug.

\section{RESULTS AND DISCUSSIONS}

Preparation and characterization of nanosuspension

Nanoprecipitation technique was used for the preparation of nanoparticles of low water-soluble ritonavir. Methanol act as a good solvent for solubilization of ritonavir as well as polymers whereas water as antisolvent. The rapid diffusion followed by evaporation of methanol from dispersed droplets into the aqueous phase lead to fast precipitation of dissolved drug within the polymers in the form of nanoparticles.

\section{Particle size analysis}

Particle size is the key parameter for efficient application of such type of formulation. Contents of polymer and surfactant showed a significant effect on particle size and its distribution. All formulations showed a mean particle size within the range of $150-328 \mathrm{~nm}$ which is well suited for the desired application (Table 2). High contents of polymer and surfactant resulted in the formation of large size particles attributed to low distribution efficiency of the internal polymer-solvent phase into the external phase. In addition, use of polymers at high content could lead to the formation of more viscous internal phase that offers resistance for mass transfer sequentially as well as diffusion of polymersolvent phase into the external phase with net effect as an increase in particle size $[14,17]$.

\section{Drug content and entrapment efficiency}

Drug content and encapsulation efficiency of nanosuspension were observed within the range of $61.15-89.59 \%$ and $40.3-93.8 \%$, respectively (Table 2), and were majorly influenced by polymer and surfactant contents. Formation of interconnected chain network between PVA and hydrophobic portions of ERL100 during precipitation could also be the key reason for the improved encapsulation efficiency $[10,18]$. 
FTIR analysis

FTIR of ritonavir (Fig. 1) showed characteristic peaks at 1645 and $1622 \mathrm{~cm}^{-1}$ (aromatic $-\mathrm{C}=\mathrm{C}$ - stretching), $1716 \mathrm{~cm}^{-1}$ (ester group), $1703.23 \mathrm{~cm}^{-1}$ (aromatic ketonic carbonyl stretching), $2964 \mathrm{~cm}^{-1}$ (acid $\mathrm{H}$-bond), and $3480 \mathrm{~cm}^{-1}$ (N-H stretching). Most of these characteristic peaks were found to be retained in the FTIR spectrum of nanoparticles (Fig. 1) which confirm there were no any structural changes in the drug. However, FTIR spectra of nanoparticles showed strong intermolecular hydrogen bonding as revealed by a strong absorption peak at $3380 \mathrm{~cm}^{-1}$ [19]. The formation of H-bond between ritonavir and polymer is the key factor for improved dissolution rate of the drug from nanoparticles than pure drug.

\section{DSC study}

DSC measures the requirement of energy by test material necessary to establish a nearly zero temperature difference between a test material and an inert reference material, as these two materials were subjected to identical temperature regimes in an environment heated or cooled at a controlled rate. A change in DSC thermogram could help to find out any possible interaction between the drug and polymer that may lead to reduced drug entrapment efficiency of the polymer as well as the altered efficacy of the drug. DSC thermogram of ritonavir (Fig. 2) showed a sharp endothermic peak at $126.95^{\circ} \mathrm{C}$ related to its melting point. However, it was very slightly shifted to $126^{\circ} \mathrm{C}$ with a broad peak in prepared nanoparticles (Fig. 2) in the presence of carrier system indicating unchanged thermal behavior of the drug in nanoparticles $[14,20,21]$.

\section{PXRD study}

PXRD was performed to study the polymorphic transformation as well as the influence of carrier on the possible phase transformation in the ritonavir in its pure form and nanoparticles. The PXRD pattern of ritonavir (Fig. 3) showed sharp and intense peaks at a diffraction angle $(2 \theta)$ of $11.73^{\circ}, 18.13^{\circ}, 18.34^{\circ}, 21.51^{\circ}, 25.30^{\circ}, 32.24^{\circ}$, and $38.61^{\circ}$ indicating high crystalline nature. However, less intense, broadened and reduced number of sharp, distinctive peaks in nanoparticles indicated a significant decrease in the degree of crystallinity of the drug and transformation into an amorphous form [14,21]. Moreover, less intense peaks of ritonavir indicated the presence of some crystalline part of drug in nanoparticles. Therefore, the reduced crystallinity of pure ritonavir in nanoparticles is the main reason for the improved rate of drug dissolution.

\section{TEM study}

From the TEM microphotographs (Fig. 4), the ritonavir loaded nanoparticles were observed as discrete, homogenous, in nanometer size range $(<50 \mathrm{~nm})$ with a smooth surface, and spherical shape without aggregation. The small size of nanoparticles obtained with nanoprecipitation method was accountable for the improved rate of drug dissolution from the nanoparticles (lyophilized nanosuspension) as well as the nanosuspension.

\section{In vitro dissolution study}

The dissolution profile of ritonavir, physical mixture, nanosuspension, and nanoparticles is shown in Fig. 5. A significant increase in the rate of dissolution, as well as complete drug release within $10 \mathrm{~min}$, was observed from nanosuspension compared to nanoparticles, physical mixture, and pure drug. This was attributed to the high rate of mass transfer due to amorphous nature and increased surface area of the drug in prepared nanosuspension. However, retarded drug release from nanoparticles compared to nanosuspension could be due to the aggregation of the particles by lyophilization of nanosuspension [22-24].

\section{In vivo bioavailability study}

In vivo bioavailability of ritonavir was estimated using male Wistar rats in a two-way parallel design. A total of 12 rats weighing 200-250 g individually were randomly and equally divided into two groups. The first group received the pure ritonavir (as reference) in suspension form
Table 2: Mean particle size, drug content, and entrapment efficiency of ritonavir nanoparticles*

\begin{tabular}{llll}
\hline Batch code & $\begin{array}{l}\text { Mean particle } \\
\text { size (nm) }\end{array}$ & $\begin{array}{l}\text { Drug } \\
\text { content (\%) }\end{array}$ & $\begin{array}{l}\text { Entrapment } \\
\text { efficiency (\%) }\end{array}$ \\
\hline RNP 1 & $195 \pm 2$ & $72.21 \pm 3.11$ & $78.7 \pm 0.21$ \\
RNP 2 & $218 \pm 9$ & $86.02 \pm 2.84$ & $83.4 \pm 0.72$ \\
RNP 3 & $328 \pm 6$ & $89.59 \pm 5.76$ & $93.8 \pm 0.18$ \\
RNP 4 & $150 \pm 4$ & $61.15 \pm 1.82$ & $40.3 \pm 0.46$ \\
RNP 5 & $179 \pm 3$ & $65.84 \pm 1.61$ & $61.6 \pm 0.83$ \\
RNP 6 & $204 \pm 1$ & $75.91 \pm 2.30$ & $80.7 \pm 0.12$ \\
\hline
\end{tabular}

*All values were expressed as the average \pm standard deviation $(n=3)$. RNP: Required navigation performance

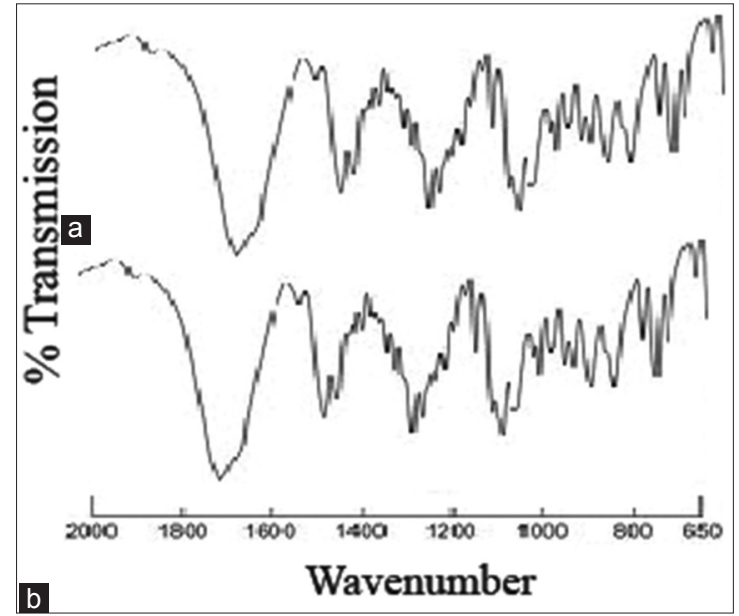

Fig. 1: Fourier transform infrared spectroscopy spectra of (a) ritonavir and (b) nanoparticles

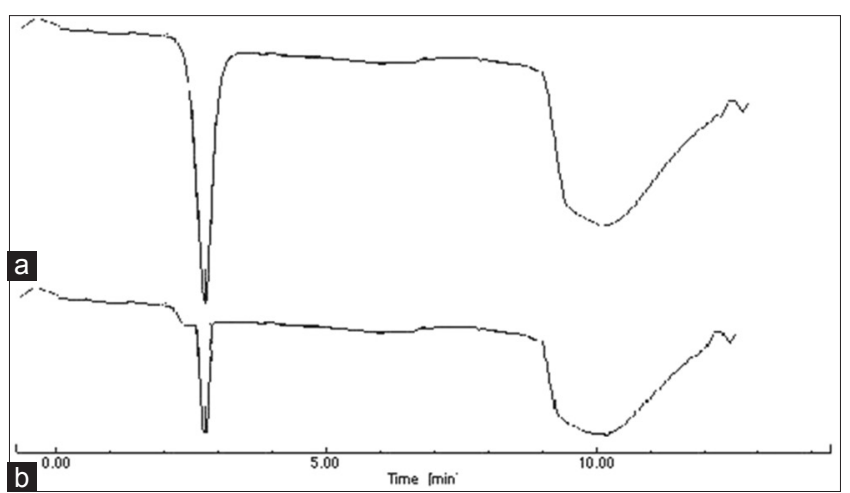

Fig. 2: Differential scanning calorimetry thermograms of (a) ritonavir and (b) nanoparticles

and the second group received the test formulation (nanosuspension). The aliquots withdrawn at predetermined time points were analyzed by the high-performance liquid chromatography method developed and validated for the estimation of ritonavir in plasma. Further, the plasma concentration-time profile of ritonavir in male Wistar rats was compared for the area under the curve (AUC), peak plasma concentration $\left(\mathrm{C}_{\max }\right)$, and time required to reach peak plasma concentration $\left(\mathrm{T}_{\max }\right)$. The $\mathrm{AUC}_{(0-48 \mathrm{~h})}$ was estimated by trapezoidal rule, and $\mathrm{AUC}_{(0-\infty)}$ was determined by the sum of the AUC and log-linear concentration divided by elimination rate constant $\left(\mathrm{K}_{\mathrm{e}}\right)[5,6]$. The elimination rate constant was determined by a linear regression analysis of log-linear part of the plasma concentration-time profile. Pharmacokinetic data obtained for ritonavir after analysis of biological samples collected from the rats are summarized in Table 3. A significant difference in $\mathrm{C}_{\max }$ and AUC was observed after comparing the mean plasma concentration-time profiles of test and reference formulations. 


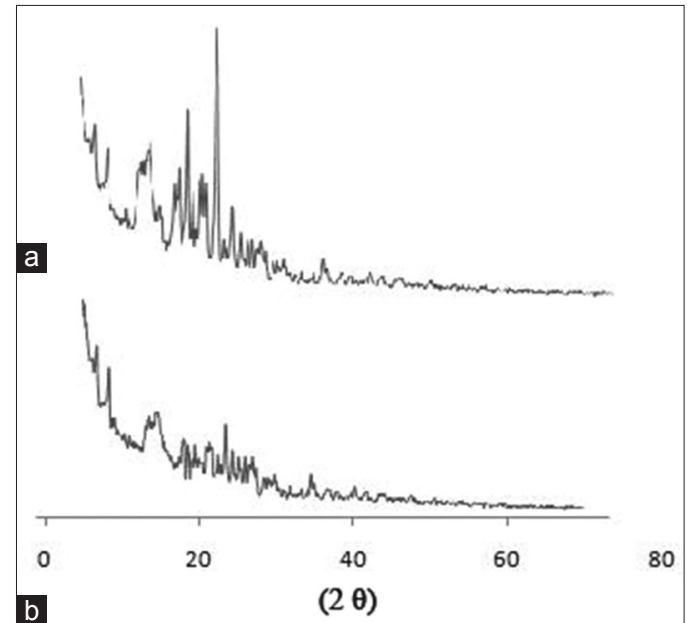

Fig. 3: Powder X-ray diffraction patterns of (a) ritonavir and (b) nanoparticles

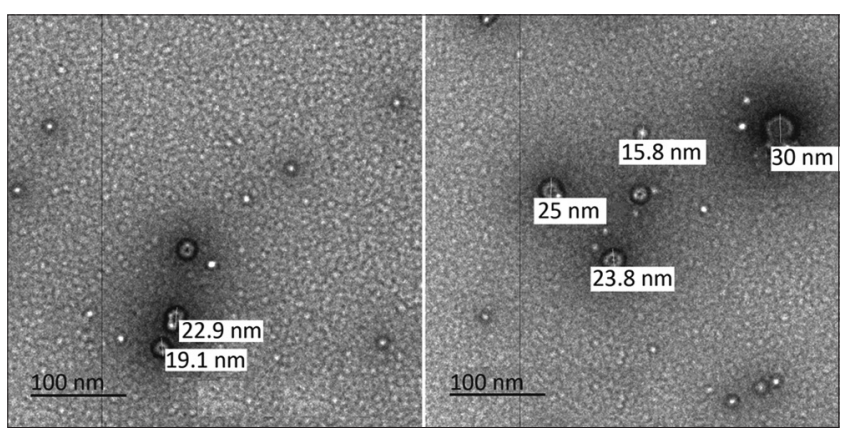

Fig. 4: Transmission electron microphotographs of ritonavir loaded nanoparticles

The nanosuspension exhibited higher bioavailability compared to the pure ritonavir. This was attributed to the improved rate of dissolution due to its amorphous nature as well as reduced diffusion layer thickness and particle size with increase in the surface area [7-11,14].

\section{CONCLUSION}

Ritonavir nanoparticulate formulation was successfully developed for improved drug dissolution as well as bioavailability by the nanoprecipitation technique with the polymers such as Eudragit RL100 and PVA. Particle size and distribution, drug content, encapsulation efficiency, and in vitro release of nanosuspension were found to be influenced by polymeric contents. Hydrophobic portions of Eudragit RL100 lead to enhancement in encapsulation efficiency of ritonavir. High polymer and surfactant contents increased the particle size proportionately. The nanosize of the ritonavir leads to the significant increase in in vitro dissolution rate with complete drug release and bioavailability as well from the nanosuspension and nanoparticles compared to pure drug and physical mixture. The nanoparticles prepared by the nanoprecipitation technique is a promising approach for the bioavailability enhancement of ritonavir and can be explored for the development of the solid dosage form for oral administration.

\section{ACKNOWLEDGMENTS}

Authors are thankful to Macleod Pharmaceutical Pvt. Ltd., Mumbai (Maharashtra, India), for gift sample of Ritonavir and Degussa Pharma. Pvt. Ltd., Mumbai (Maharashtra, India), for the kind gift of ERL100 and PVA.

\section{REFERENCES}

1. Amidon GL, Lennernäs H, Shah VP, Crison JR. A theoretical basis for a biopharmaceutic drug classification: The correlation of in vitro

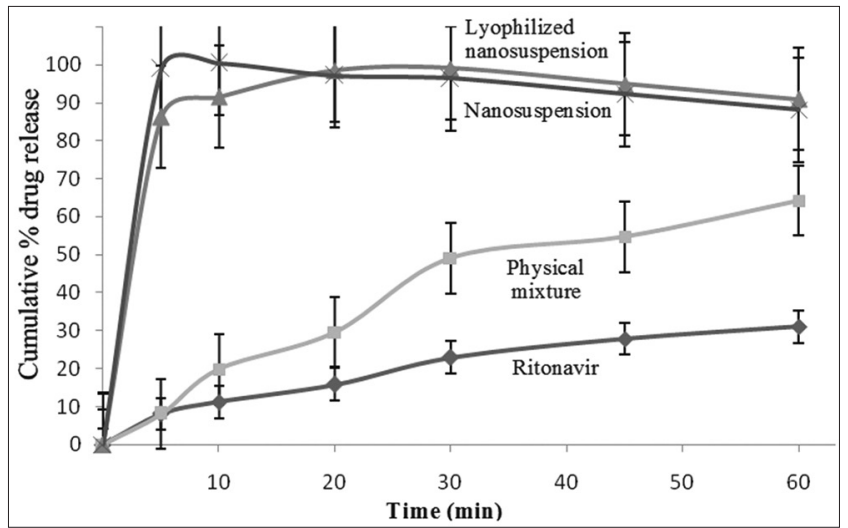

Fig. 5: The dissolution profile of ritonavir, physical mixture, nanosuspension, and nanoparticles

Table 3: Pharmacokinetic data of ritonavir estimated from in vivo bioavailability study

\begin{tabular}{|c|c|c|}
\hline Parameter & Reference & Test \\
\hline $\mathrm{C}_{\max }(\mathrm{ng} / \mathrm{ml})$ & 1359.5 & 21127.22 \\
\hline $\mathrm{T}_{\max }^{\max }(\mathrm{h})$ & 3 & 0.5 \\
\hline $\mathrm{AUC}_{(0.48 \mathrm{~h})}(\mathrm{ng} . \mathrm{h} / \mathrm{ml})$ & 2060.24 & 14496.6 \\
\hline $\mathrm{AUC}^{(0-48 \mathrm{~h})}$ (ng.h/ml) & 2072.41 & 14510.1 \\
\hline Elimination rate constant $\left(\mathrm{K}_{\mathrm{e}}\right)\left(\mathrm{h}^{-1}\right)$ & 0.302 & 0.406 \\
\hline Half life (h) & 2.3 & 1.7 \\
\hline
\end{tabular}

drug product dissolution and in vivo bioavailability. Pharm Res 1995;12:413-20.

2. Dahan A, Miller JM, Amidon GL. Prediction of solubility and permeability class membership: Provisional BCS classification of the world's top oral drugs. AAPS J 2009;11:740-6.

3. Benet LZ, Amidon GL, Barends DM, Lennernäs H, Polli JE, Shah VP, et al. The use of BDDCS in classifying the permeability of marketed drugs. Pharm Res 2008;25:483-8.

4. Hadinoto K, Zhu K, Tan RB. Drug release study of large hollow nanoparticulate aggregates carrier particles for pulmonary delivery. Int J Pharm 2007;341:195-206.

5. Breitenbach J. Melt extrusion: From process to drug delivery technology. Eur J Pharm Biopharm 2002;54:107-17.

6. Kawakami K, Yoshikawa T, Moroto Y, Kanaoka E, Takahashi K, Nishihara $\mathrm{Y}$, et al. Microemulsion formulation for enhanced absorption of poorly soluble drugs. I. Prescription design. J Control Release 2002;81:65-74.

7. Davis ME, Brewster ME. Cyclodextrin-based pharmaceutics: Past, present and future. Nat Rev Drug Discov 2004;3:1023-35.

8. Gullapalli RP. Soft gelatin capsules (softgels). J Pharm Sci 2010;99:4107-48.

9. Sethia S, Squillante E. Solid dispersions: Revival with greater possibilities and applications in oral drug delivery. Crit Rev Ther Drug Carrier Syst 2003;20:215-47.

10. Davis SS, Washington C, West P, Illum L, Liversidge G, Sternson L, et al. Lipid emulsions as drug delivery systems. Ann N Y Acad Sci 1987;507:75-88

11. Sham JO, Zhang Y, Finlay WH, Roa WH, Löbenberg R. Formulation and characterization of spray-dried powders containing nanoparticles for aerosol delivery to the lung. Int J Pharm 2004;269:457-67.

12. Mahalingam M, Krishnamoorthy K. Camptothecin loaded poly (methacyclic acid-co-methyl-methyacrylate) nanoparticles: Fabrication, characterization and cytotoxicity studies. Int J Pharm Pharm Sci 2015;7:135-40.

13. Mishra R, Mir SR, Amin S. Polymeric nanoparticles for improved bioavailability of cilnidipine. Int J Pharm Pharm Sci 2017;9:129-39.

14. Sinha S, Ali M, Baboota S, Ahuja A, Kumar A, Ali J. Solid dispersion as an approach for bioavailability enhancement of poorly water-soluble drug ritonavir. AAPS Pharm Sci Technol 2010;11:518-27.

15. Parboosing R, Maguire GE, Govender P, Kruger HG. Nanotechnology and the treatment of HIV infection. Viruses 2012;4:488-520.

16. Devi K, Pai R. Antiretrovirals: Need for an effective drug delivery. 
Indian J Pharm Sci 2006;68:1

17. Fessi C, Devissaguet JP, Puisieux F, Thies C. Process for the Preparation of Dispersible Colloidal Systems of a Substance in the form of Nanoparticles. US Patent No. 5118528 A; 1990.

18. Pouton CW. Lipid formulations for oral administration of drugs: Non-emulsifying, self-emulsifying and "self-microemulsifying" drug delivery systems. Eur J Pharm Sci 2000;11 Suppl 2:S93-8.

19. Bilati U, Allémann E, Doelker E. Development of a nanoprecipitation method intended for the entrapment of hydrophilic drugs into nanoparticles. Eur J Pharm Sci 2005;24:67-75.

20. Vetrichelvan T, Sowkar BI. Formulation and in vitro characterization of sustained release ritonovir nanoparticles in alginates. J Pharm Res
2011;44:2152-5

21. Hancock BC, Zografi G. Characteristics and significance of the amorphous state in pharmaceutical systems. J Pharm Sci 1997;86:1-12.

22. Leuner C, Dressman J. Improving drug solubility for oral delivery using solid dispersions. Eur J Pharm Biopharm 2000;50:47-60.

23. Serajuddin AT. Solid dispersion of poorly water-soluble drugs: Early promises, subsequent problems, and recent breakthroughs. J Pharm Sci 1999;88:1058-66.

24. Merisko-Liversidge E, Liversidge GG. Nanosizing for oral and parenteral drug delivery: A perspective on formulating poorly-water soluble compounds using wet media milling technology. Adv Drug Deliv Rev 2011;63:427-40. 